\title{
ZNF804A risk allele is associated with relatively intact gray matter volume in patients with schizophrenia
}

\author{
Gary Donohoe a,c,*,1, Emma Rose ${ }^{\mathrm{a}, 1}$, Thomas Frodl ${ }^{\mathrm{a}, \mathrm{b}, \mathrm{c}}$, Derek Morris ${ }^{\mathrm{a}}$, Ilaria Spoletini ${ }^{\mathrm{d}}$, \\ Fulvia Adriano $^{\mathrm{d}}$, Sergio Bernardini ${ }^{\mathrm{e}}$, Carlo Caltagirone ${ }^{\mathrm{d}, \mathrm{f}}$, Paola Bossù ${ }^{\mathrm{d}}$, Michael Gill ${ }^{\mathrm{a}, \mathrm{c}}$, \\ Aiden P. Corvin ${ }^{\mathrm{a}, \mathrm{c}}$, Gianfranco Spalletta ${ }^{\mathrm{d}}$
}

a Neuropsychiatric Genetics Research Group, Trinity College Dublin, Dublin, Ireland

b Clinical Neuroimaging Research Group, Department of Psychiatry, Trinity College Dublin, Dublin, Ireland

c Trinity College Institute for Neuroscience, Trinity College Dublin, Ireland

d IRCCS Santa Lucia Foundation, Dept of Clinical and Behavioural Neurology, Via Ardeatina, 306-00179 Rome, Italy

e Department of Internal Medicine and Laboratory Medicine, Tor Vergata University, Rome, Italy

${ }^{\mathrm{f}}$ Department of Neuroscience, Tor Vergata University, Rome, Italy

\section{A R T I C L E I N F O}

\section{Article history:}

Received 28 June 2010

Revised 9 September 2010

Accepted 27 September 2010

Available online 8 October 2010

\section{Keywords:}

Schizophrenia

MRI

Voxel-based morphometry

ZNF804A

\begin{abstract}
A B S T R A C T
ZNF804A rs1344706 is the first genetic risk variant to achieve genome wide significance for psychosis. Following earlier evidence that patients carrying the ZNF804A risk allele had relatively spared memory function compared to patient non-carriers, we investigated whether ZNF804A was also associated with variation in brain volume. In a sample of 70 patients and 38 healthy participants we used voxel based morphometry to compare homozygous (AA) carriers of the ZNF804A risk allele to heterozygous and homozygous (AC/CC) non-carriers for both whole brain volume and specific regions implicated in earlier ZNF804A studies-the dorsolateral pre-frontal cortex, the hippocampus, and the amygdala. For patients, but not for controls, we found that homozygous 'AA' risk carriers had relatively larger gray matter volumes than heterozygous/homozygous non-carriers ( $\mathrm{AC} / \mathrm{CC}$ ), particularly for hippocampal volumes. These data are consistent with our earlier behavioral data and suggest that ZNF804A is delineating a schizophrenia subtype characterized by relatively intact brain volume. Establishing if this represents a discrete molecular pathogenesis with consequences for nosology and treatment will be an important next step in understanding ZNF084A's role in illness risk.
\end{abstract}

(c) 2010 Elsevier Inc. All rights reserved.

\section{Introduction}

A single nucleotide polymorphism rs1344706 located at gene ZNF804A (OMIM: 612282) was the first genetic variant to achieve genome wide significance for psychosis (9.96X10-9 (OR 1.12)) (O'Donovan et al., 2008a,b). Despite being relatively under-powered to replicate such a modest effect, two of three recently reported large SZ GWAS studies (Purcell et al., 2009; Sullivan et al., 2008) and a discrete replication study by Riley et al. (2009) supported association between the same risk allele and increased SZ risk. Copy number variation at this locus has also been identified for psychosis (Steinberg et al., 2010). The protein encoded by ZNF804A is uncharacterized, but given its zinc finger domain, is likely to have a role in regulating gene expression. The mouse orthologue Zfp804A is widely expressed in the brain (Allen brain atlas (http://www.brain-map.org/); known binding partners include ataxin-1, encoded by ATXN1 (OMIM: 601556).

\footnotetext{
* Corresponding author. Clinical Psychology, Department of Psychiatry, Trinity Health Centre, St. James's Hospital, Dublin 8, Ireland. Fax: + 35318962467. E-mail address: donoghug@tcd.ie (G. Donohoe).

${ }^{1}$ Both authors contributed equally to this work.
}

Changes in cognitive function and brain structure are strongly associated with SZ and are predictive of level of disability (Green et al., 2004; Toulopoulou et al., 2007). Investigating the functional contribution of individual SZ risk variants to these changes has the unique advantage of enabling in vivo investigation of candidate genes at the level of brain and behavior. The utility of this approach has been demonstrated by findings with existing candidate genes for schizophrenia (including DISC1, NRG1, DTNBP1, DAOA (G72), RGS4; discussed in O'Donovan et al., 2008a,b). Recently, Esslinger et al. (2009) reported that the ZNF804A risk 'A' allele was associated with altered connectivity in the dorsolateral pre-frontal cortex (DLPFC) and the hippocampus. They found that connectivity between the hippocampus and DLPFC was increased in risk carriers, while connectivity between contralateral DLPFC regions and within the right DLPFC was reduced. The authors speculated the reduced connectivity in DLPFC may be a contributory factor in disturbed executive function, whereas the increased connectivity between DLPFC and hippocampus might lead to a disruption of interactions between prefrontal and limbic cortices. However, it is pertinent to note that this study only included healthy controls and no cognitive correlations with these connectivity effects were observed. A recent study by our group, involving both patients and healthy 
Table 1

Participant characteristics.

\begin{tabular}{|c|c|c|c|}
\hline & Schizophrenic patients $(N=70)$ & Healthy controls $(N=38)$ & Comparison \\
\hline Age (years; mean (s.d.)) & $40.44(11.70)$ & $32.49(12.74)$ & $t_{(106)}=3.09, p=0.003$ \\
\hline Years of education (mean (s.d.)) & $10.71(4.11)$ & $15.38(3.20)$ & $t_{(84)}=-5.40, p<0.001$ \\
\hline Gender (male/female) & $46: 24$ & $20: 18$ & $\chi^{2}=1.77, d f=1, \mathrm{NS}$ \\
\hline ZNF804 rs1344706 genotype (CC/AC/AA) & $13: 30: 27$ & $5: 14: 19$ & $\chi^{2}=1.41, d f=2, \mathrm{NS}$ \\
\hline Age at onset (years; mean (s.d.)) & $24.10(8.18)$ & - & $\mathrm{N} / \mathrm{A}$ \\
\hline Duration of illness (mean (s.d.)) & $17.82(10.59)$ & - & $\mathrm{N} / \mathrm{A}$ \\
\hline Chlorpromazine equivalents (mg/day; mean (s.d.)) & $500.60(588.62)$ & - & $\mathrm{N} / \mathrm{A}$ \\
\hline
\end{tabular}

participants, suggested an alternative explanation. We found that the risk allele at ZNF804A was associated with relatively spared cognitive ability in the domains of working and episodic memory in independent samples of Irish and German patients with SZ but not controls (Walters et al., 2010). Of note, these cognitive abilities involve two of the three brain regions-the dorsolateral prefrontal cortex and hippocampusassociated with ZNF804A in the Esslinger study (Smith and Jonides, 1999; Squire et al., 2004). We further demonstrated that the association between ZNF804A and SZ increased when patients with IQs lower than 90 were excluded from the analysis. Collectively, these results suggest that ZNF804A is not associated with cognitive impairments, and appears instead to delineate an illness subtype in which cognitive deficits are not a strong feature.

Cognitive deficits have been repeatedly correlated with reduced gray matter volume in SZ (Hulshoff Pol et al., 2006). Based on our earlier cognitive study we predicted that ZNF804A risk A allele carriers would show relatively larger gray matter volumes than non-carriers either at the whole brain level or in a planned region of interest analyses of brain regions implicated by Esslinger et al. (2009) (the dorsolateral prefrontal cortex (DLPFC), the hippocampus, and the amygdala), and our behavioral study (the DLPFC and hippocampus). Avoiding sample bias, we undertook this study in a relatively large sample of patients who were independent of the sample included in our original cognitive analysis.

\section{Materials and methods}

\section{Participants}

All participants gave written informed consent for this study, in accordance with local ethics committee guidelines. Structural magnetic resonance imaging (MRI) was conducted and samples for genotyping were acquired from 82 patients and 39 controls. Twelve patients and one control participant were subsequently excluded from data analysis because of issues relating the scan quality (e.g. head motion) or genotyping (e.g. insufficient DNA volumes), resulting in a final sample of 70 patients and 38 controls. Patients were recruited consecutively from two outpatient clinics in central Italy, and all imaged on the same scanner. Exclusion criteria for patients recruited were: (1) any axis I or II psychiatric disease except schizophrenia; (2) a history of traumatic brain injury with a loss of consciousness; (3) a history of epilepsy or seizures, or relevant neurological or medical illness (e.g. cerebrovascular disease) and (4) history of substance abuse in the 6 months preceding the study. Healthy participants were recruited from the same geographic area as patients and were screened for current or past diagnosis of any axis I or II disorder using the SCID-I and SCID-II (First et al., 1997; First et al., 2002). In addition to the exclusion criteria outlined above, an exclusion criterion for healthy controls was a history of schizophrenia or any other mental disorder diagnosis among firstdegree relatives. All participants were Caucasian and were of Italian ancestry (Table 1 ).

\section{Genotyping}

The samples were genotyped using a Taqman ${ }^{\circledR}$ SNP Genotyping Assay on a 7900HT Sequence Detection System (Applied Biosystems).
The call rate for the Taqman genotyping was $100 \%$ and the samples were in Hardy-Weinberg Equilibrium $(p>0.05)$. Along with the Italian samples, a number $(n=10)$ of HapMap CEU DNA samples (http://www.hapmap.org) were genotyped for rs1344706 for quality control purposes. All genotypes were found to be concordant with available online HapMap data. Twenty seven patients were identified as homozygous for the 'A' risk allele of the ZNF804A polymorphism, 30 as heterozygous, and 13 as homozygous for the ' $C$ ' allele. The control group consisted of 19 ' $A$ ' allele homozygous, 14 heterozygous/ 'AC', and 5 ' $C$ ' homozygous individuals.

\section{MRI acquisition}

The magnetic resonance images were acquired at the IRCCS Fondazione Santa Lucia in Rome, Italy, using a $1.5 \mathrm{~T}$ whole-body Siemens Vision Magnetom scanner. T2 and fluid attenuated inversion recovery (FLAIR) sequences were acquired to screen for brain pathology. For voxel-based morphometry analyses, a T1-weighted 3D data set was acquired using a magnetization-prepared rapid gradient echo sequence $(\mathrm{TR}=11.4 \mathrm{~ms}, \mathrm{TE}=4.4 \mathrm{~ms}$, flip angle $=151$, slice thickness $=1 \mathrm{~mm}$, no inter-slice gap, voxel size $=1 \times 1 \times 1 \mathrm{~mm})$. Data pre-processing were performed using SPM5 (Wellcome Department of Cognitive Neurology, London, UK http://www.fil.ion.ucl.ac.uk/spm) running under MATLAB (version 7.8; The MathWorks, Cambridge, UK).

\section{Voxel based morphometry (VBM; Ashburner and Friston, 2000)}

Data analysis was performed using the VBM toolbox (version 5.1; http://www.dbm.neuro.uni-jena.de/vbm) for SPM5. Individual volumes were checked for scanner artefacts and gross anatomical abnormalities, and then segmented into gray matter (GM), white matter (WM), and cerebrospinal fluid (CSF), using the Hidden Markov Random Field (HMRF; weighting 0.15) option in VBM 5.1 to minimize noise. Segmented images were then normalized using the DARTEL toolbox (Ashburner, 2007). Templates for GM and WM were created using default parameters for DARTEL template creation. Jacobian scaled ("modulated") warped tissue classes were then created for both GM and $\mathrm{WM}$, and the resultant images were then smoothed with an $8 \times 8 \times 8 \mathrm{~mm}$ Gaussian kernel.

\section{Statistical analysis}

The impact of ZNF804A genotype was considered using full factorial ANOVA in SPM5, and included both diagnosis (patient vs. control) and genotype as fixed factors. Given the relatively low number of homozygous non-risk ' $C$ ' allele individuals, and the lack of a dose-dependent effect of the ' $A$ ' risk allele in our previous behavioral results (Walters et al., 2010), these analyses focused on a comparison of homozygous AA risk genotype carriers versus carriers of one or two copies of the ' $C$ ' non-risk allele (i.e. a combined AC/CC genotype group). Two whole brain analyses were conducted-one for GM and one for white matter. In order to control for between-group differences, both age and gender were included as co-variates in these analyses. Furthermore, to account for normal variation in brain volume, total gray or white matter volume for each subject was also 
included as a co-variate. Interaction and simple effects of group and genotype were considered at $p_{\text {UCNORRECTED }}<0.001$ and $p_{\text {CORRECTED }}<0.05$. As a consequence of issues of non-uniform smoothness in VBM data (Ashburner and Friston, 2000; Worsley et al., 1999), prior to interpretation of results a non-stationarity cluster extent correction was implemented using the NS toolbox for SMP5 (http://www.fmri. wfubmc.edu/cms/NS-General), which utilized the random field theory version of cluster inference under non-stationarity (Hayasaka et al., 2004).

Given previous functional imaging and behavioral evidence suggesting a role for ZNF804A in areas of the brain associated with memory (e.g. episodic or working memory), we supplemented our whole brain analyses with two a priori region of interest (ROI) analyses i.e.: (1) bilateral DLPFC; and (2) bilateral hippocampus and amygdala. These analyses were performed using the WFU pickatlas toolbox version 2.4 for SPM5 (Maldjian et al., 2003). In pickatlas, regions for each of the ROI analyses were selected from a predefined list of Brodmann areas (BA). For the DLPFC ROI analysis bilateral BA9 and BA46 were selected, whereas the second ROI included the entire hippocampus and amygdala, bilaterally. As with our whole-brain analyses, both interaction and simple effects of group and genotype were considered for each $\mathrm{ROI}$ and a non-stationarity correction was applied to the data. Following correction for multiple comparisons, which accounted for the entire volume under consideration (i.e. bilateral DLPFC or bilateral hippocampus/amygdala), those regions which were significant at $p_{\text {CORRECTED }}<0.05$ and exceeded a minimum cluster extent of 100 voxels $\left(100 \mathrm{~mm}^{3}\right)$ were considered to be significant.

\section{Results}

\section{Main effect of participant group}

Significant reductions in GM volume were noted in patients, compared to controls, in a number of regions including superior and middle temporal and middle and medial superior frontal gyri, inferior parietal lobes, insula, thalamus and cingulate gyrus (see Supplemental Fig. S1 and Supplemental Table S1 for an abbreviated list of regions that survived correction for multiple comparisons at the whole-brain level). While a number of regions of white matter demonstrated a similar main effect of participant group, none of these clusters survived correction for multiple comparisons.

Post-hoc analyses of each of the GM ROIs under consideration (i.e. DLPFC and hippocampus/amygdala) were also indicative of a significant (i.e. $p_{\text {CORRECTED }}<0.05$ ) effect of group in all sub-regions, such that GM volume was greater in controls.

\section{ZNF804A and clinical and demographic differences}

Demographic characteristics of patients and healthy controls grouped according to ZNF804A genotype are outlined in Table 2. No genotype related differences in age, gender, or years in education were observed for either sample. For the patient group no between-genotype differences in illness onset, duration, medication dosage (in chlorpromazine equivalents) or symptom severity (measured using the positive and negative symptom rating scale; Kay et al., 1988) were observed.

\section{ZNF804A whole brain analysis}

For the whole brain VBM analysis, GM and WM volume did not vary as a function of ZNF804A genotype. However, there was a significant (i.e. $p_{\text {UNCORRECTED }}<0.001$ ) interaction between ZNF804A genotype and diagnosis (i.e. patient vs. healthy participant) in a number of brain regions, including temporal and prefrontal areas (see Table S2 for details). Post hoc analysis revealed that this interaction was the consequence of a number of regions of relatively greater GM volume for homozygous carriers of the ZNF804A risk ' $\mathrm{A}$ ' allele, compared to ' $C$ ' allele carriers, in patients only (see Fig. $1 \mathrm{~A}$ and $\mathrm{B}$ and Supplemental Table S3). Areas of increased volume in homozygous risk carriers included the bilateral superior temporal gyrus and insula-however, no region survived correction for multiple comparisons $\left(p_{\mathrm{FWE}}<0.05\right)$. There were no regions which showed the opposite effect for patients (i.e. $\mathrm{AC} / \mathrm{CC}>\mathrm{CC}$ ) and no regions of significantly different volume for control participants only, irrespective of the direction of difference being tested (i.e. $A A>A C / C C$ or $A C / C C>A A$ ).

\section{ZNF804A region of interest (ROI) analysis}

Two separate, planned GM ROI analyses considered the impact of ZNF804A genotype on (1) bilateral DLPFC (including BA46 and BA9) and (2) bilateral hippocampus and amygdala. A significant interaction between group and genotype was noted in the hippocampal/amygdala ROI (i.e. right hippocampus; MNI co-ordinates $32-9-11$; see Fig. 1C), whereas there were no significant effects of genotype and no significant interactions between genotype and group in DLPFC. Following correction for multiple comparisons (see 'Materials and methods' for details), we determined that this interaction was the consequence of a patient-specific variation in GM volume associated with ZNF804A genotype. Homozygous risk 'A' allele carriers had significantly larger hippocampal volume compared to heterozygous and homozygous carriers of the non-risk ' $C$ ' allele $\left(K_{\mathrm{E}}=179\right.$ voxels; $t_{(102)}=3.72$, $p_{\text {CORRECTED }}=0.032$; Cohen's $d=0.74$ ).

A post-hoc simple effects analysis of the impact of genotype on brain volume in the hippocampal/amygdala ROI was carried out for patients only. In addition, to replicating the previous observation, this analysis was also indicative of significantly greater volume in a cluster in the left hemisphere (i.e. MNI co-ordinates $-32-12-13$; $\left(t_{(102)}=3.59\right.$, $K_{\mathrm{E}}=159$ voxels, $p_{\text {CORRECTED }}=0.01$; Cohen's $d=0.71$ ).

To determine whether or not patient characteristics contributed to the observed effect on hippocampal volume, we considered whether there were any significant differences between genotype groups with

Table 2

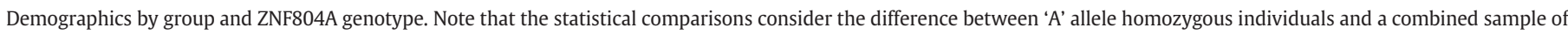
one or two C copy carriers.

\begin{tabular}{|c|c|c|c|c|c|c|c|c|}
\hline & \multicolumn{4}{|l|}{ Cases } & \multicolumn{4}{|l|}{ Controls } \\
\hline & $\mathrm{AA}(N=27)$ & $\mathrm{AC}(N=30)$ & $\mathrm{CC}(N=13)$ & Comparison & $\mathrm{AA}(N=19)$ & $\mathrm{AC}(N=14)$ & $\mathrm{CC}(N=5)$ & Comparison \\
\hline Age (years: mean (s.d.)) & $40.36(11.59)$ & $42.71(11.89)$ & $42.36(11.86)$ & $t_{(68)}=1.03$ N.S. & $32.05(13.07)$ & $31.93(13.58)$ & $38.80(10.04)$ & $t_{(36)}=0.4$ N.S. \\
\hline Gender (\% Female) & 24.32 & 36.67 & 30.77 & $\chi^{2}=0.16$ N.S. & 36.84 & 71.43 & 20.00 & $\chi^{2}=1.69$ df N.S. \\
\hline Education (years: mean (s.d.)) & $10.72(4.47)$ & 11.35 (3.79) & $9.00(4.17)$ & $t_{(65)}=-.01 \mathrm{~N} . \mathrm{S}$. & $15.87(2.75)$ & $13.55(2.38)$ & 19.67 (3.79) & $t_{(27)}=-0.8$ N.S. \\
\hline Age at onset & $22.32(7.11)$ & $25.29(9.84)$ & $25.27(6.62)$ & $t_{(59)}=1.43$ N.S. & & & & \\
\hline Duration of illness & $18.02(9.91)$ & $17.42(11.16)$ & $17.09(11.64)$ & $t_{(59)}=-.13$ N.S. & & & & \\
\hline Panss positive & $25.38(8.50)$ & $23.63(6.52)$ & $24.79(6.5)$ & $t_{(55)}=-.37$ N.S. & & & & \\
\hline Panss negative & $28.38(9.1)$ & $22.47(7.79)$ & $26.18(8.6)$ & $t_{(55)}=-.92$ N.S. & & & & \\
\hline Panss general & $60.77(10.90)$ & $53.3(13.20)$ & $55.96(12.9)$ & $t_{(55)}=-.13$ N.S. & & & & \\
\hline Chlorpromazine equivalents & $448.52(249.55)$ & $605.46(871.51)$ & $390.18(318.16)$ & $t_{(58)}=0.58$ N.S. & - & - & - & - \\
\hline
\end{tabular}


A

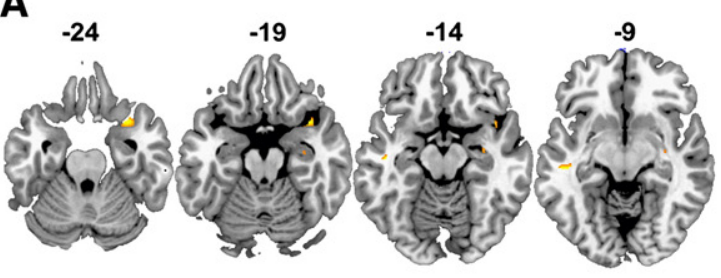
हो 1 (1)

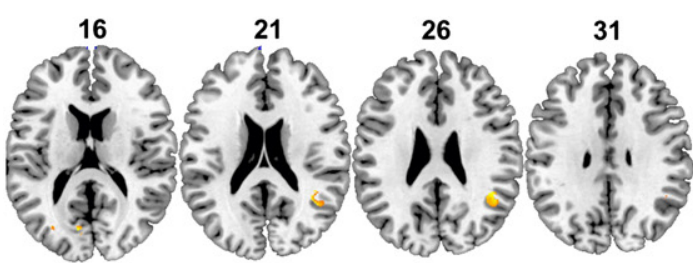

B

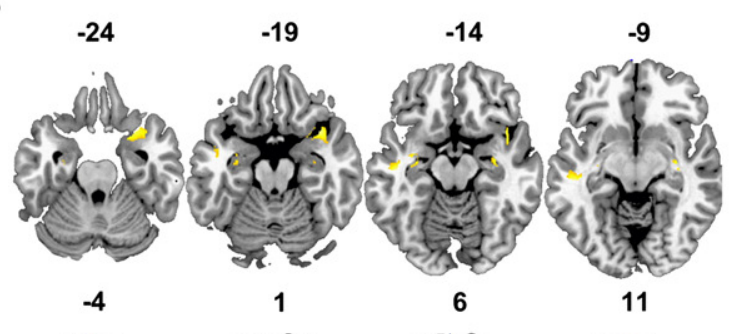

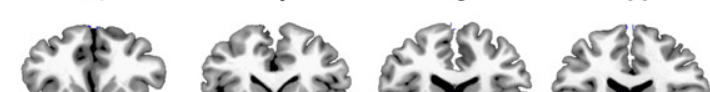

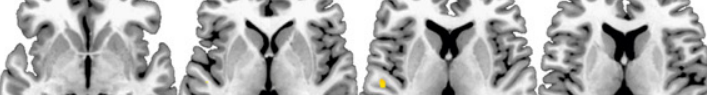
Eल

2

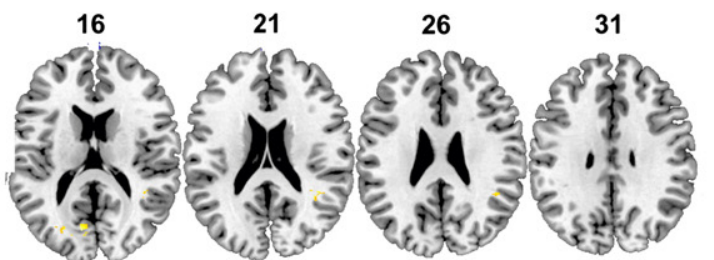

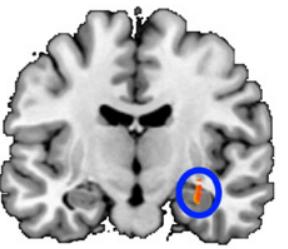

32
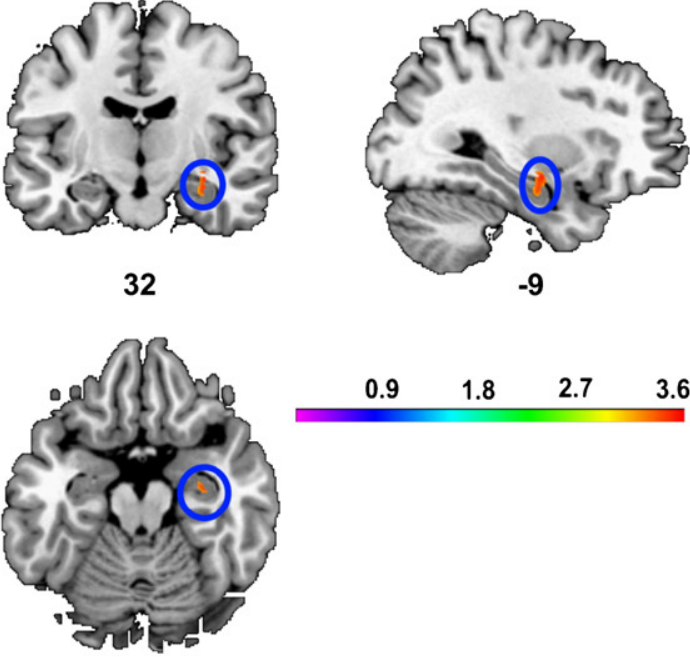

\begin{tabular}{llll}
0.9 & 1.8 & 2.7 & 3.6 \\
\hline
\end{tabular}

$-11$

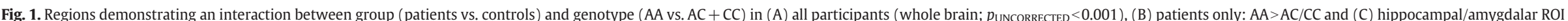
$\left(p_{\text {CORRECTED }}<0.05\right)$ analyses of GM in schizophrenic patients only. Note: intensity bars represent the statistical value $(F$ or $t)$ at a given voxel. 
regards to age at illness onset, duration of illness and current dosage of chlorpromazine equivalents. ZNF genotype in patients was not associated with any of these factors. Furthermore, the inclusion of duration of illness as a co-variate in the whole brain and ROI analysis of VBM in patients did not alter the pattern of results described above.

\section{Discussion}

The rs1344706 risk 'A' allele located within ZNF804A, the first variant showing genome wide evidence of association with psychosis, has previously been associated with altered connectivity between brain regions including the DLPFC, hippocampus and amygdala in healthy controls (Esslinger et al., 2009), and with relatively fewer cognitive impairments in episodic and working memory function in patients (Walters et al., 2010). In the present study a priori ROI analysis found that the same variant was associated with relatively higher right hippocampal gray matter volume in patients only. The direction of these results was consistent with our whole brain analysis-which implicated the superior temporal gyrus and insula bilaterally-although these differences did not survive correction for multiple testing.

The protein encoded by ZNF804A is uncharacterized, but given its zinc finger domain is hypothesized to regulate gene expression. A recent study by Riley et al (2009) found that in a combined sample of patients and controls stratified according to the ' $\mathrm{A}$ ' risk allele, risk carriers showed increased gene expression relative to non-carriers. These data provide evidence of the functional importance of either this SNP or other variants in linkage disequilibrium (LD) with it, although the mechanism by which SZ risk is being elevated is unclear. SZ has consistently been associated with poorer cognitive function, yet this ZNF804A variant has been associated with spared cognitive ability relative to other patient non-carriers (Walters et al., 2010). The findings of the present studythat, for patients, carrying the ZNF804A risk ' $\mathrm{A}$ ' allele is associated with relatively greater gray matter and hippocampal volumes than noncarriers-again falls in the same direction.

Relatively increased expression, relatively spared cognitive performance, and (in the present study) relatively higher gray matter volume appear counter-intuitive for a SZ risk variant. We have previously argued that this apparent advantage may result from ZNF804A's delineating a particular SZ subtype in which cognitive impairment is a less prominent feature (Walters et al., 2010). When compared to patients who are non-carriers (and whose disease pathway is presumably associated with other more cognitively damaging risk factors) the risk associated with ZNF804A emerges as a relative advantage. Given the association between cognitive ability and gray matter volume (Hulshoff Pol et al., 2006), the association with higher gray matter volume is consistent with this interpretation. Moreover, the functionally related cognitive and cortical overlap indicated by these findings is also consistent with this explanation-the association with the hippocampus in the present study corresponds closely to the association with memory function in our behavioral data. Finally, it is noteworthy that all of the samples included here were independent of those included in our earlier behavioral findings. While the results from both datasets are consistent (e.g. in implicating memory function and hippocampal volume), further study would benefit from each of these measures being available with the same sample.

In interpreting our data the absence of an association between ZNF804A genotype and brain volume in healthy participants is noteworthy. One possible reason may have been insufficient power given the smaller number of healthy volunteers, a shortcoming of our study. However, since completing this study we have repeated the analysis in an unrelated sample of healthy Irish controls $(N=140)$ who had undergone functional imaging and had been genotyped for ZNF804A. Again, no effect of ZNF804A genotype was observed for these healthy controls in either whole brain or ROI analysis based on either an AA vs. AC/CC groups analysis or on the basis of individual genotype groups (i.e. $\mathrm{AA}=49$; $A C=68 ; C C=23$ ) considered separately (data available upon request).
ZNF804A's relevance to cortical functioning in healthy controls is supported by two recent studies. Esslinger et al. (2009) observed altered functional connectivity both bilaterally between dorsolateral prefrontal cortex (DLPFC), and between DLPFC, temporal lobes, and amygdala. In a second study by the same group using an overlapping dataset, Walter et al. (2010) also found differences in medial prefrontal and left temporo-parietal cortical activations during a theory of mind task, again in healthy controls. While the cortical regions implicated in that study overlap with those associated with ZNF804A in the present study, how these functional effects might be expected to relate to structural changes in gray matter volume is unclear given the apparent differences in the direction of the findings. Interpreting these differences is complicated by the fact that these studies focused only on healthy controls and did not include patients with psychiatric illness. Given the possibility that a genetic variant may have different biological effects in patients and controls due to differences in the background load of other risk variants present in each group, clarifying whether the effects of ZNF804A are similar or different remains an important issue for understanding the biological role of this variant.

\section{Conclusions}

$\mathrm{SZ}$ is a heterogeneous disorder, not just in terms of clinical symptomatology, but also in level of cognitive disability. It is probable therefore that not all genetic risk factors increase illness liability via a deleterious impact either on cognitive function or the brain structures serving these functions. Together with previously reported neuropsychological data from our group, this study suggests that ZNF804A may be delineating an illness subtype characterized by relatively intact memory function and relatively spared hippocampal structure in patients. In doing so, cognitive neuroscience approaches such as those reported here may be contributing to a molecular nosology of SZ, defined both by identified genetic risk factors and their specific cortical effects.

\section{Appendix A. Supplementary data}

Supplementary data to this article can be found online at doi:10.1016/j.neuroimage.2010.09.089.

\section{References}

Ashburner, J., 2007. A fast diffeomorphic image registration algorithm. NeuroImage 38 (1), 95-113.

Ashburner, J., Friston, K.J., 2000. Voxel-based morphometry-the methods. NeuroImage 11 (6), 805-821.

Esslinger, C., Walter, H., Kirsch, P., Erk, S., Schnell, K., Arnold, C., et al., 2009. Neural mechanisms of a genome-wide supported psychosis variant. Science 324 (5927), 605-605.

First, M.B., Gibbon, M., Spitzer, R.L., Williams, J.B.W., Benjamin, L.S., 1997. Structured Clinical Interview for DSM-IV Axis I Disorders-Clinical Version (SCID-CV). American Psychiatric Press, Inc., Washington, D.C.

First, M.B., Spitzer, R.L., Miriam, G., Willaims, J.B.W., 2002. User's Guide for the Structured Clinical Interview for DSM-IV Axis I Disorders-Clinical Version (SCID-CV). Biometrics Research, New York State Psychiatric Institute, New York.

Green, M.F., Kern, R.S., Heaton, R.K., 2004. Longitudinal studies of cognition and functional outcome in schizophrenia: implications for MATRICS. Schizophr. Res. 72 (1), 41-51.

Hayasaka, S., Phan, K.L., Liberzon, I., Worsley, K.J., Nichols, T.E., 2004. Nonstationary cluster-size inference with random field and permutation methods. Neurolmage 22 (2), 676-687.

Hulshoff Pol, H.E., Schnack, H.G., Mandl, R.C., Brans, R.G., van Haren, N.E., Baare, W.F., et al, 2006. Gray and white matter density changes in monozygotic and same-sex dizygotic twins discordant for schizophrenia using voxel-based morphometry. Neurolmage 31 (2), 482-488.

Kay, S.R., Opler, L.A., Lindenmayer, J.P., 1988. Reliability and validity of the Positive and Negative Syndrome Scale for schizophrenia. Psychiatry Res. 23, 99-110.

Maldjian, J.A., Laurienti, P.J., Kraft, R.A., Burdette, J.H., 2003. An automated method for neuroanatomic and cytoarchitectonic atlas-based interrogation of fMRI data sets. NeuroImage 19 (3), 1233-1239.

O'Donovan, M.C., Craddock, N., Norton, N., Williams, H., Peirce, T., Moskvina, V., et al., 2008a. Identification of loci associated with schizophrenia by genome-wide association and follow-up. Nat. Genet. 40 (9), 1053-1055. 
O'Donovan, M.C., Craddock, N., Owen, M.J., 2008b. Schizophrenia: complex genetics, not fairy tales. Psychol. Med. 38 (12), 1697-1699.

Purcell, S.M., Wray, N.R., Stone, J.L., Visscher, P.M., O'Donovan, M.C., Sullivan, P.F., et al., 2009. Common polygenic variation contributes to risk of schizophrenia and bipolar disorder. Nature 460 (7256), 748-752.

Riley, B., Thiselton, D., Maher, B.S., Bigdeli, T., Wormley, B., McMichael, G.O., et al., 2009. Replication of association between schizophrenia and ZNF804A in the Irish CaseControl Study of Schizophrenia sample. Mol. Psychiatry 15 (1), 29-37.

Smith, E.E., Jonides, J., 1999. Neuroscience-storage and executive processes in the frontal lobes. Science 283 (5408), 1657-1661.

Squire, L.R., Stark, C.E.L., Clark, R.E., 2004. The medial temporal lobe. Annu. Rev. Neurosci. 27, 279-306.

Steinberg, S., Mors, O., Borglum, A.D., Gustafsson, O., Werge, T., Mortensen, P.B., et al. 2010. Expanding the range of ZNF804A variants conferring risk of psychosis. Mol. Psychiatry (epub ahead of printing).
Sullivan, P.F., Lin, D., Tzeng, J.Y., van den Oord, E., Perkins, D., Stroup, T.S., et al., 2008. Genomewide association for schizophrenia in the CATIE study: results of stage 1. Mol. Psychiatry 13 (6), 570-584.

Toulopoulou, T., Picchioni, M., Rijsdijk, F., Hua-Hall, M., Ettinger, U., Sham, P., et al., 2007. Substantial genetic overlap between neurocognition and schizophrenia-genetic modeling in twin samples. Arch. Gen. Psychiatry 64 (12), 1348-1355.

Walter, H., Schnell, K., Erk, S., Arnold, C Kirsch, P., Esslinger, C., et al., 2010. Effects of a genome-wide supported psychosis risk variant on neural activation during a theory-of-mind task. Mol. Psychiatry (epub ahead of printing).

Walters, J.T.R., Corvin, A., Williams, H., Dragovic, M., Quinn, E. M., Judge, R., et al., 2010. The psychosis susceptibility gene ZNF804A is associated with less impaired cognitive performance in schizophrenia. Arch. Gen. Psychiatry 67 (7), 692-700.

Worsley, K.J., Andermann, M., Koulis, T., MacDonald, D., Evans, A.C., 1999. Detecting changes in nonisotropic images. Hum. Brain Mapp. 8 (2-3), 98-101. 